\title{
Microtensile Bond Strength of Cad-Cam and Pressed-Ceramic Inlays to Dentin
}

\author{
A. Nilgün Öztürka, DDS, PhD \\ Özgür İnana, DDS, PhD \\ Erkan İnan ${ }^{b}$, \\ Bora Öztürkc , DDS, PhD
}

\section{ABSTRACT}

Objectives: CAD-CAM system is popular because of high esthetic and short fabrication time. But, there is limited information available about the microtensile bonding of luting cements to CAD-CAM inlays and to dentin. The aim of this study was to examine the bond strength of CAD-CAM (Cerec 3) and pressed-ceramic (IPS Empress 2) inlays to dentin surface by microtensile testing using two luting cements.

Materials and Methods: Standardized mesio-occlusal cavities were made in forty extracted molar teeth. An occlusal reduction of $2 \mathrm{~mm}$ was made; the bucco-lingual width of the proximal boxes was 4 $\mathrm{mm}$, the occlusal width $3 \mathrm{~mm}$ and the depth of the pulpal and axial walls $2 \mathrm{~mm}$. The proximal boxes were extended $1 \mathrm{~mm}$ below the cemento-enamel junction. Teeth were randomly assigned to 2 groups to evaluate the bonding of 2 ceramic systems, Cerec 3 (Group I) and IPS Empress 2 (Group II), to dentin. Each of the 2 groups were further divided into 2 luting cement groups, Panavia F (Group A) and Variolink II (Group B). After cementation, the teeth were sectioned into two $1.2 \times 1.2 \mathrm{~mm}$ wide 'I' shape sections. The specimens were then subjected to microtensile testing at a crosshead speed of $1 \mathrm{~mm} / \mathrm{min}$. Twoway ANOVA and Tukey HSD tests were used to evaluate the results.

Results: The mean microtensile bond strengths of Cerec 3 and IPS Empress 2 bonding to dentin with

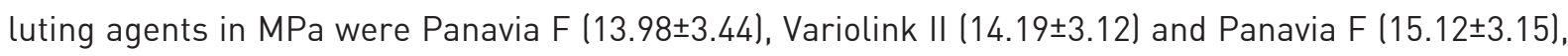
Variolink II (15.45 \pm 3.08 ) respectively. No significant differences were found among the 2 ceramic systems $(P>.05)$ and 2 luting cements with regard to dentin bond strengths $(P>.05)$.

Conlusions: There was no difference found between the dentin bond strength of the Cerec 3 and IPS Empress 2 inlays cemented with two luting cements. (Eur J Dent 2007;2:91-96)

Key Words: Dentin; Bond strength; Resin cements; Inlay; CAD-CAM; IPS Empress.

\section{INTRODUCTION}

An increasing interest in aesthetics during the past decade has resulted in the development of a great number of tooth-colored inlay systems as an

$\square$ a Selcuk University, School of Dentistry, Department of Prosthodontics, Konya, Turkey.

b Private Practice in Tafers, Switzerland.

Selcuk University, School of Dentistry,

Department of Operative Dentistry, Konya, Turkey.

$\square$ Corresponding author: Dr. A. Nilgün ÖZTÜRK

Selcuk University, Department of Prosthodontics,

Faculty of Dentistry Campus / Konya, Turkey.

Phone: +90 33224100 41/1186 Fax: +90332 2410062

E-mail: nilgun25ahotmail.com alternative to silver amalgam alloys in particular in posterior teeth. ${ }^{1}$ Ceramic inlays are viable alternatives to composite inlays with regard to the disadvantages of composite material, such as wear, polymerization shrinkage and high coefficient of thermal expansion. ${ }^{2}$

Since the end of the $19^{\text {th }}$ century when ceramic inlays were first described several new materials and techniques for the construction of indirect esthetic restorations have been introduced. ${ }^{2}$ For ceramic inlays manually manufactured in dental 
laboratories a commonly used technique is the heat-pressed IPS Empress. Heat-press system utilizes the lost-wax technique., ${ }^{3,4}$ Thus this technique requires an impression and a model of the prepared tooth. These procedures may be eliminated by using CAD-CAM system. ${ }^{5}$ The Cerec CADCAM system offers the opportunity to prepare, design, and fabricate a ceramic restoration in a single appointment, without the need for making impressions, provisional restorations, or dental laboratory support. ${ }^{6}$

Besides the advantages of the indirect ceramic, bonding to the tooth structure is still a challenging matter. In order to establish a strong and durable bond, which is necessary for the biomechanical aspect of the tooth-restoration system, appropriate treatment of the respective surfaces is crucial. ${ }^{7,8}$ With contemporary adhesive cements and the new generation of bonding systems, achieving a strong and durable bond to the tooth structure and the indirect restoration could be feasible. ${ }^{8}$ Current ceramic bonding systems are based on mechanochemical bonding between the luting material and ceramic restorations. The application of silane couplers served as the chemical surface preparation for bonding porcelain. The use of silane primers considerably enhanced the bond strength of porcelain. ${ }^{9-13}$

Several investigations have been carried out to test the bond strength of resin cements to ceramics using the different conventional test methods. But, there is limited information available about the microtensile bonding performance of resin cements to Cerec 3 ceramic inlays. Microtensile test method is different, therefore it may result in better estimates of the bond strength of a specimen. ${ }^{14-18}$ The purpose of this study was to investigate the bond strength of CAD-CAM (Cerec 3) and pressed-ceramic (IPS Empress 2) inlays to dentin surface by microtensile testing using two luting cements.

\section{MATERIALS AND METHODS}

Forty human molars, extracted for periodontal reasons and free of caries or restorations, were selected for this study. The teeth were cleaned and stored in saline during the study. Teeth were embedded up to $2 \mathrm{~mm}$ from the cemento-enamel junction into autopolymerizing resin (Bayer Ltd., Newbury, United Kingdom).

\section{Preparation design}

Standardized mesio-occlusal Class II cavities were made with round burs (No:6801L.314, Gebr. Brasseler, Lemgo. Germanyl and 6-degree conical diamond burs (No:8959KR.314.016; Gebr. Brasseler) in a high-speed handpiece mounted on a parallelometer (Bego Bremer Goldschagerei Wihl. Herbst Gmbh \& Co., Bremen, Germany). An occlusal reduction of $2 \mathrm{~mm}$ was made; the buccolingual width of the proximal boxes was $4 \mathrm{~mm}$, the occlusal width $3 \mathrm{~mm}$ and the depth of the pulpal and axial walls $2 \mathrm{~mm}$. The proximal boxes were extended $1 \mathrm{~mm}$ below the cemento-enamel junction. No bevels were utilized in the preparation.

The 40 prepared teeth were randomly assigned to 2 groups of 20 , each to 2 ceramic systems, Cerec 3 (Sirona, A.G., Bensheim, Germany) (Group I) and IPS Empress 2 (Ivoclar, Schaan, Liechtenstein) (Group II). Each of the 2 groups were further divided into 2 luting cement groups, Panavia F (Kuraray Medical Inc., Kurashiki, Okayama, Japan) (Group A) and Variolink II (Ivoclar Vivadent, Schaan, Liechtenstein) (Group B).

\section{Fabrication of inlay restorations}

Impressions were made of teeth preparations with vinyl polysiloxane impression material (Permagum, 3M ESPE AG, Seefeld, Germany) and poured in a vacuum mixed polyurethane die material (Alpha Die MF, Schültz-Dental GmbH, Rosbach, Germanyl according to the manufacturer's instructions. Ceramic inlays were pressed. All procedures were performed with IPS Empress 2 materials and protocol.

The Cerec 3 ceramic inlays were manufactured in accordance with designing the inlay with computer-aided manufacturing methods. The inlay cavities were coated by spraying a thin layer of Vita Cerec powder (Cerec Propellant, Vita Zahnfabrik, Bad Sackingen, Germany). The orientation of the camera was carefully adjusted until all cavity margins could clearly be recognized on the two dimensional image of the preparation. The optical impression of the preparation was then recorded and the full three-dimensional frame of the restoration was designed and machined with CEREC 3 CAD-CAM system (Sirona, A.G., Bensheim, Germanyl. The inlays were cut from Vitablocks Mark II CEREC porcelain of the shade-A2 I8 (Vita Zahnfabrik, Bad Sackingen, Germanyl. 


\section{Luting Procedures}

Both IPS Empress 2 and Cerec 3 ceramic inlays were luted in the same way. In Group IA, IIA; Panavia $F$ dual polymerizing resin cement was used. Ceramic inlays were etched with phosphoric acid gel (K Etchant, Kuraray Co., Ltd. Osaka, Japan) for 5 seconds. A layer of silane coupling agent combination (Clearfil Porcelain Bond Activator, Clearfil Liner Bond 2V Primer; Kuraray Co., Ltd.) was applied to the ceramic bonding surfaces for 5 seconds, then air-dried. Panavia F ED, the selfetching primer was applied to the dentin surface for 60 seconds and gently air-dried. Panavia F was mixed for 20 seconds and applied to the bonding surface of the ceramic restorations. Finger pressure was used to stabilize the inlays during to the dentin surface. Excessive cement was removed with an explorer and the cement was polymerized for 20 seconds for occlusal surface with the light polymerizing unit at $350 \mathrm{~mW} / \mathrm{cm}^{2}$ (with the light tip to specimen distance of $0 \mathrm{~mm}$ ) (Hilux 350, Express Dental Products, Toronto, Canada). The margins were covered with glycerin jelly for 3 minutes and washed.

In Group IB and IIB, Variolink II was used. The ceramic inlays were treated with hydrofluoric acid (Ceramic Etchant, Ceramco, Burlington, NJ) for 1 minute and neutralized (Ceramic Etchant Neutralizer, Ceramcol in accordance with the manufacturer's instructions. Silane (Monobond S, Ivoclar) was first applied with a brush to the ceramic inlays for 60 seconds and then bonding agent (Heliobond, Ivoclar) was applied. After the teeth were etched, primer (Syntac Primer, Ivoclar) was applied to the tooth surface for 15 seconds, adhesive (Syntac Adhesive, Ivoclar) for 10 seconds, and then bonding agent (Heliobond, Ivoclar) with a brush. Cement (Variolink II, Vivadent, Ivoclar), comprising a combination of $50 \%$ Variolink yellow base, and $50 \%$ catalyst was hand-mixed following the manufacturer's directions, and applied to both prepared teeth and the ceramic inlays. The ceramic inlays were seated on the teeth with finger pressure and excess cement was removed with an explorer. Light polymerization was performed with the light polymerizing unit at $350 \mathrm{~mW} / \mathrm{cm}^{2}$ (with the light tip to specimen distance of $0 \mathrm{~mm}$ ) for 40 seconds for occlusal surface. After cementation, specimens were stored in distilled water at $37^{\circ} \mathrm{C}$ for 24 hours and then thermocycled for 500 cycles between $5{ }^{\circ} \mathrm{C}$ and $55^{\circ} \mathrm{C}$ using a dwell time of 30 seconds.

\section{Microtensile testing}

Acrylic blocks were fixed to the mounting plate of a slow-speed diamond saw sectioning machine. The roots were removed from the remaining crown approximately $2 \mathrm{~mm}$ below the cemento-enamel junction. Each tooth was vertically sectioned both mesial-distally and buccal-lingually along their long axis into $1.2 \times 1.2 \mathrm{~mm}$ wide sections. Two ' shape sectioned longitudinal cuts, the top half consisting of ceramic and the bottom half consisting of dentin, were made from each tooth (Figure $1 A, B)$. Therefore, there were 20 specimens per group and a total of 80 specimens were subjected to tensile forces.

These specimens were then attached to the microtensile testing apparatus (Harvard Apparatus Co. Inc., Dover, Mass.) with cyanoacrylate adhesive (Zapit, Dental Ventures of America, Corona, CA, USA). The specimens were then subjected to tensile forces at a crosshead speed of $1 \mathrm{~mm} / \mathrm{min}$, and the maximum load at fracture $(\mathrm{Kg})$ was re-

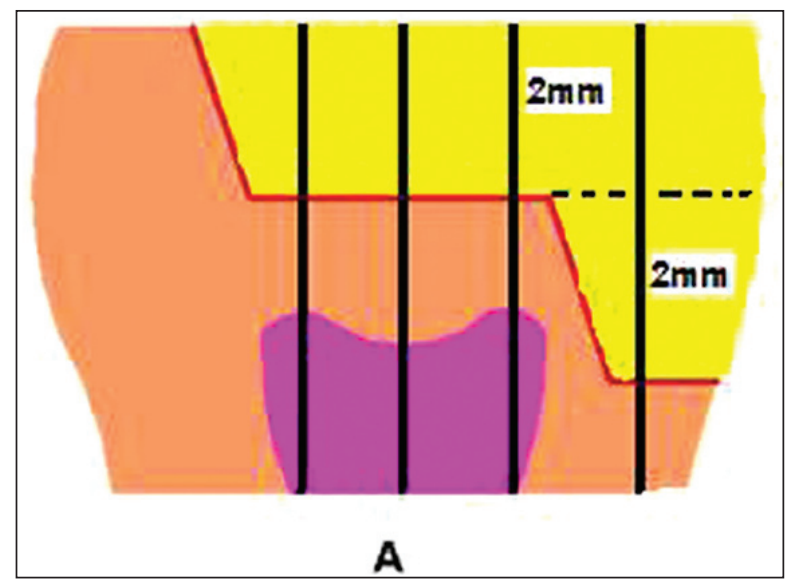

Figure 1A. Specimen preparation for microtensile bond test.

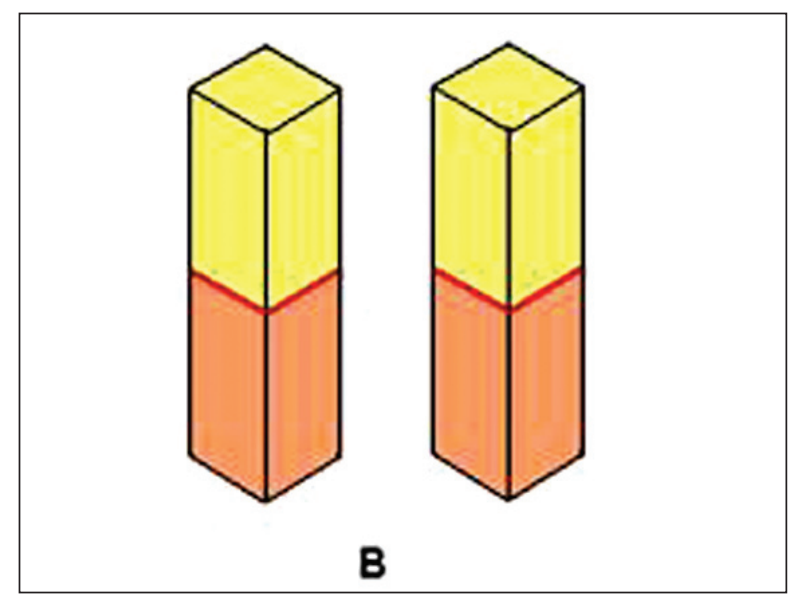

Figure 1B. I-shape sections for microtensile bond test. 
corded. The values were converted to MPa. Preparation of all specimens and completion of the testing were done by the same operator.

After the specimens fractured, the fractured surfaces were observed using a stereomicroscope (SZ-TP, Olympus, Tokyo, Japan) at 22x magnification to identify the mode of fracture. The fractured surface was classified according to 1 of 3 types:

Type 1: Adhesive failure between luting cement and dentin.

Type 2: Cohesive failure in the luting cement.

Type 3: Cohesive failure in the dentin.

\section{SEM examination}

To observe the interface, the teeth were sectioned mesiodistally through the restoration. The specimens were first polished with 240, 300 and 600 -grit silicon carbide adhesive paper. The polished interface was etched for 30 seconds using $10 \%$ phosphoric acid, rinsed for 30 seconds, and $10 \% \mathrm{NaOCl}$ applied to the surface for 5 minutes, rinsed for 30 seconds, and then dried thoroughly. Specimens were sputter-coated (Leo 435 VP, Cambridge, England) with gold and interfaces observed under SEM (Leo 435 VP, Cambridge, England).

The statistical analysis was performed using SPSS for windows 2000/V 10.0 ISPSS Inc, Chicago,

Table 1. Results of 2-way analysis of variance of microtensile bond strength.

\begin{tabular}{lccccc}
\hline Source & df & $\begin{array}{c}\text { Sum of } \\
\text { squares }\end{array}$ & $\begin{array}{c}\text { Mean } \\
\text { square }\end{array}$ & $\begin{array}{c}F \\
\text { value }\end{array}$ & $\begin{array}{c}P \\
\text { value }\end{array}$ \\
$\begin{array}{lccccc}\text { Ceramicx luting } \\
\text { cement }\end{array}$ & 1 & $7.2 \times 10^{-2}$ & $7.2 \times 10^{-2}$ & 0.007 & 0.934 \\
Ceramic & 1 & 28.8 & 28.8 & 2.802 & 0.098 \\
Luting cement & 1 & 1.404 & 1.404 & 0.137 & 0.713 \\
\hline
\end{tabular}

Table 2. Mean $\pm S D$ tensile bond strength values (MPa) for experimental groups.

\begin{tabular}{lccc}
\hline Groups & $\begin{array}{c}\text { Ceramic/Luting } \\
\text { cement }\end{array}$ & Number & Mean \pm SD \\
I A & $\begin{array}{c}\text { CEREC 3, Panavia F } \\
\text { IPS Empress 2, }\end{array}$ & 20 & $13.98 \pm 3.44$ \\
II A & $\begin{array}{c}\text { Panavia F } \\
\text { I B }\end{array}$ & 20 & $15.12 \pm 3.15$ \\
& CEREC 3, Variolink II & 20 & $14.19 \pm 3.12$ \\
II B & $\begin{array}{c}\text { IPS Empress 2, } \\
\text { Variolink II }\end{array}$ & 20 & $15.45 \pm 3.08$ \\
\hline
\end{tabular}

IL, USA). The bond strength values were analyzed with 2-way analysis of variance (ANOVA) and Tukey HSD tests. Differences were considered significant at $\mathrm{P}<.05$.

\section{RESULTS}

2-way ANOVA indicated that microtensile bond strength was not significantly affected by luting cements ( $P>$.05) and ceramic systems ( $P>$.05) (Table 1). The means, standard deviations of the 2 luting cements of the bond strengths for each ceramic system are given in Table 2. There was no difference found between the dentin bond strength of the Cerec 3 and IPS Empress 2 ceramic inlays cemented with two different luting cements.

Most failures (72 of 80 ) were adhesive at the luting cement/dentin interface (Type 1). 8 specimens showed cohesive failure within the luting cement (Type 2). Two of these cohesive failures were seen in Group IA, 3 were seen in Group IB, 3 were seen in Group IIA.

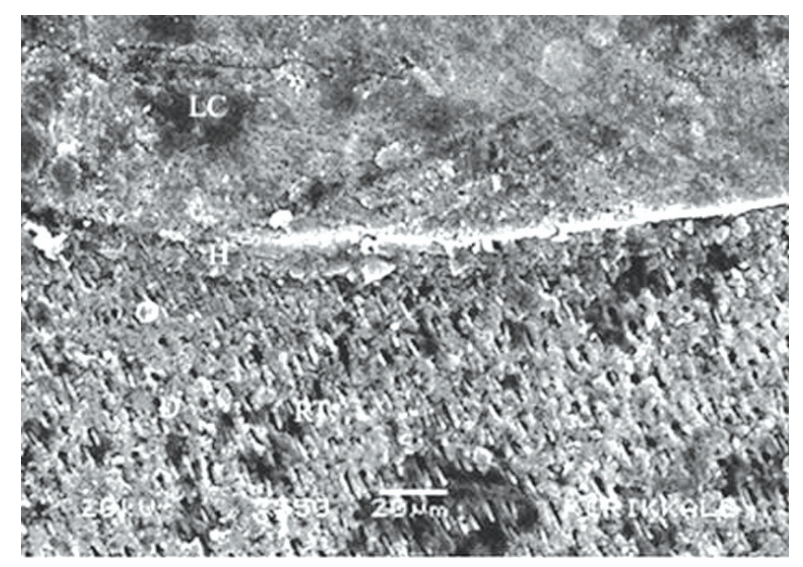

Figure 2. SEM view of demineralized specimen for Panavia F. (D:Dentin, LC:Luting Cement, RT:Resin Tags, H:Hybrid layer) (X550)

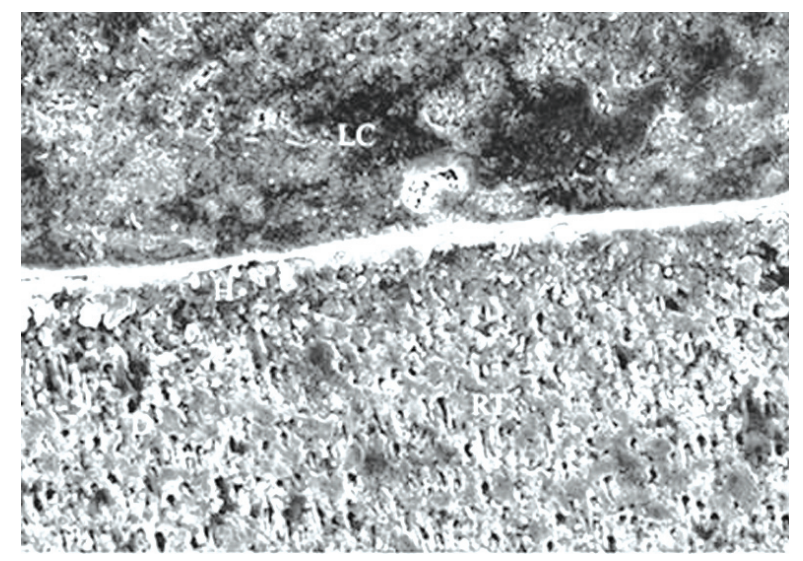

Figure 3. SEM view of demineralized specimen for Variolink II. (D:Dentin, LC:Luting Cement, RT:Resin Tags, H:Hybrid layer) (X500) 
In SEM evaluation, the formation of resin tags and non-uniform hybrid layer were evidenced in both luting cements (Figures 2 and 3).

\section{DISCUSSION}

A strong, durable bonding can influence the longevity of the ceramic restoration, improves marginal adaptation and prevents microleakage and increases fracture resistance of the restored tooth and the restoration. ${ }^{7,8}$ An increasing number of all-ceramic materials and systems are currently available for inlays and onlays. Examples of new manufacturing techniques introduced in dentistry are the CAD-CAM technique and the commonly used pressable ceramics. ${ }^{4}$ In this study, microtensile bond strength of the Cerec 3 CAD-CAM system and IPS Empress II pressable ceramic system which were luted with Panavia F and Variolink II was evaluated.

The Cerec method for restoring teeth by CADCAM is now over 10 years old. ${ }^{6}$ The clinical performance of restorations machined by a CAD-CAM system is determined by the total "process chain". Three main factors may determine this. Firstly operator variables expressed as both clinical skills and expertise with the CEREC machine. Secondly, the intrinsic limitations of hardware devices such as the data-acquisition camera and the milling unit. Finally, the software program and the design algorithms it uses will determine the accuracy of the proposed restoration design. CAD-CAM milling of feldspathic ceramic blocks fabricated under controlled and optimum manufacturer conditions enables the production of a restoration with a higher intrinsic strength without the material variations inevitable in laboratory produced restorations. ${ }^{5}$ Leucite-reinforced feldspathic porcelain, IPS Empress offers higher strength for both anterior and posterior teeth if resin bonding techniques are properly applied. These materials are brittle and fracture easily, so the use of adhesive resins to bond to sound tooth structure enhances the longevity of these restorations. ${ }^{19}$ Polymerization shrinkage is another characteristic of resins. ${ }^{20}$ To ensure a tight seal between the tooth surface and ceramic, the use of strong adhesive resins is also recommended. ${ }^{19,20}$ When comparing the microtensile bond strength of Cerec 3 inlays to IPS Empress 2 inlays, no differences were found among the ceramic systems and two different dual polymerizing cements in this study.

Bonding resin to a ceramic surface, whether porcelain or glass ceramic, is based on the combined effects of micromechanical interlocking and chemical bonding. Porcelain surfaces are generally roughened with an acid, air abrasion (sandblasting) or a diamond bur, to increase the surface area and create microporosities. The adhesive resin flows into the porosities and interlocks, forming strong micromechanical bonds. ${ }^{13}$ This study used two types of etching agents for the ceramic surfaces, K-etchant gel which is $37 \%$ phosphoric acid (Panavia F), and 4.9\% Hydrofluoric acid (Variolink II). Llobell et al $^{9}$ found significantly higher bond strengths with hydrofluoric acid compared with phosphoric acid and advised use of hydrofluoric acid for mechanical retention and silane coupling agents for chemical retention. But, in this presented study, the treatment of ceramic surfaces phosphoric or hydrofluoric acid did not cause any significant difference in bond strength values of luting cements. The published reports ${ }^{10,12}$ indicated that the chemical bonding effected by the silane primers on the porcelain surface is an important factor in improving bond strength between luting cement and porcelain. Especially, the effectiveness of the Clearfil Porcelain bond silane bonding agent (Panavia F) has been reported by several researchers. ${ }^{9.11}$ The effectiveness of the Clearfil Porcelain Bond material may be attributed to the fact that the material contained an initiator for enhancing conversion of the monomer component at the adhesive interface, and the fact that the material also contained a polymerizable hydrophobic acid catalyst.

Uno et $\mathrm{al}^{17}$ evaluated the microtensile bond strength of Cerec 2 inlays to dentin surface using three luting cements (Clapearl DC, AP-X, Fluorocement). Similar to our study, the results of this study showed that there was no significant difference in microtensile bond strength among the luting cements.

Shear and tensile strength tests are the most widely used for the bond strength measurements. Sano et $\mathrm{al}^{14}$ introduced a microtensile test method, which used a bonded surface area of approximately $1 \mathrm{~mm}^{2}$. Using this test method, Sano et $\mathrm{al}^{14}$ showed that more adhesive failures occurred at the interface, and also recorded higher bond strengths than test methods which used large 
surface areas. The microtensile test produced far more failures within the adhesive than the shear bond strength test. Thus the microtensile method may result in better estimates of the true bond strength of a material. ${ }^{15-18}$

Microtensile testing evaluates the bond capability of the restorative systems to dentin. ${ }^{14}$ The small size of the specimens has led to a more favorable stress distribution, and thus the failure of bonds which are closer to their true ultimate strengths. ${ }^{16,18}$ Therefore, in this study microtensile test method was used to evaluate bond strength of the specimens. Most of the failures were adhesive in all test groups, only 8 of them were cohesive. These results are agreement with other previously published studies. ${ }^{16,18}$

\section{CONCLUSIONS}

Dentin bond strength of the Cerec 3 and IPS Empress 2 ceramic inlays cemented with two different luting cements was similar. Long-term follow-up bond strength studies are necessary for assessment of the clinical advantages and disadvantages of the various manufacturing ceramic materials and cements.

\section{REFERENCES}

1. Sertgöz A, Gemalmaz D, Alkumru H, Yoruç B. Luting composite thickness of two ceramic inlay systems. Eur J Prost Rest Dent 1995;4:151-154.

2. Qualtrough AJ, Wilson $\mathrm{NH}$, Smith GA. Porcelain inlay: a historical view. Oper Dent 1990;15:61-70.

3. Dong JK, Luthy H, Wohlwend A, Scharer P. Heat-pressed ceramics: technology and strength. Int $J$ Prosthodont 1992;5:9-16

4. Addi S, Hedayati-Khams A, Poya A, Sjögren G. Interface gap size of manually and CAD/CAM manufactured ceramic inlays/onlays in vitro. $J$ Dent 2002;30:53-58.

5. Martin N, Jedynakiewicz NM. Clinical performance of Cerec ceramic inlays: a systematic review. Dent Mater 1999;15:54-61.

6. Mormann WH, Brandestini M, Lutz F. The Cerec system: computer-assisted preparation of direct ceramic inlays in 1 setting. Quintessence Int 1987;38:457-470.

7. Jensen ME, Sheth JJ, Tolliver D. Etched porcelain resin bonded full veneer crowns: in vitro fracture resistance. Compend 1989;10:336-338.

8. Dietschi D, Maeder M, Meyer JM, Holz J. In vitro resistance to fracture of porcelain inlays bonded to tooth. Quintessence Int 1990;21:823-830.
9. Llobell A., Nicholls JI, Kois JC, Daly CH. Fatigue life of porcelain repair systems. Int J Prosthodont 1992;5:205-213.

10. Russell DA, Meiers JC. Shear bond strength of resin composite to Dicor treated with 4-META. Int $J$ Prosthodont $1994 ; 7: 7-12$

11. Kato $H$, Matsumura $H$, Tanaka T, Atsuta M. Bond strength and durability of porcelain bonding systems. J Prosthet Dent 1996;75:163-168.

12. Kamada K, Yoshida K, Atsuta M. Effect of ceramic surface treatments on the bond of four resin luting agents to a ceramic material. J Prosthet Dent 1998;79:508-514.

13. Schwartz RS, Summitt JB,. Robbins JW, Santos JD. Fundamentals of Operative Dentistry. Quintessence Publishing Co, 2001, Inc. USA.

14. Sano H, Shono T, Sonoda H, Takatsu T, Ciucchi B, Carvalho $\mathrm{R}$ et al. Relationship between surface area for adhesion and tensile bond strength: Evaluation of a microtensile bond test. Dent Mater 1994;10:236-240.

15. Schreiner RF, Chappell RP, Glaros AG, Eick JD. Microtensile testing of dentin adhesives. Dent Mater 1998;14:194201.

16. Phrukkanon S, Burrow MF, Tyas MJ. The influence of cross-sectional shape and surface area on the microtensile bond test. Dent Mater 1998;14:212-221.

17. Uno S, Tanaka T, Kawamoto C, Konishi J, Sano H. Microtensile bond strength to dentin and cavity adaptation of Cerec 2 inlay restoration. Am J Dent 2000;13:59-63.

18. Tanumiharja M, Burrow MF, Tyas MJ. Microtensile bond strengths of seven dentin adhesive systems. Dent Mater 2000;16:180-187.

19. Inokoshi S, Van Meerbeek B, Willems G, Lambrechts $P$, Braem M, Vanherle G. Marginal accuracy of CAD/CAM inlays made with the original and the updated software. $J$ Dent 1992:20:171-177.

20. Hickel R, Kunzelmann KH, Nkenke E, Petschelt A. In Bond strength of five bonding composite resins to CerecCeramic, Quintessence, Chicago (1991), 567-575. 